\title{
Stemness Markers in Ovarian Cancer: Nature and Implications
}

\section{Amal Abd El hafez*}

Department of Pathology, Faculty of Medicine, Mansoura University, Egypt

Ovarian cancer is the fourth most common cause of cancer-related death in women and is the most lethal gynecological malignancy with $30 \%$ to $40 \%$ overall survival (OS) at 5 years [1]. Due to the non-specific symptoms and inadequate screening methods at the early stages, more than $60 \%$ of ovarian cancer patients are diagnosed at advanced stage [2]. Clinically, ovarian cancer is characterized by an initial response to combined cytoreductive surgery and chemotherapy. Subsequently, recurrence and disease progression ensue contributing to the poor patient outcome.

These observations have drawn the attention to a subset of cancer cells that initially gives rise to the tumor, gets spared after therapy, has a protracted lifespan, and displays the potential to regenerate and undergo massive proliferation and differentiation to give rise to the phenotypically and functionally more mature cancer cells.

Lately, evaluation of the cellular composition of epithelial ovarian cancers identified at least two distinct subtypes of cells: type II ovarian cancer cells which are the classical cancer cells, characterized by small size and fast cell division; and the slowly dividing type I ovarian cancer cells, that share many markers with pluripotent stem cells and have the potential to give origin to the fast-dividing type II cells [3]. Literature addressed these cells as "cancer stem cells" or "tumor initiating cells" (CSCs/TICs), and extensive research has been executed in order to define unique markers that can be used to isolate ovarian CSCs/TICs and testify their potentiality for therapeutic targeting. So, a substantial number of stem cell markers including CD44, CD117, CD133, CD24, Bmi-1, ABCG2 and aldehyde dehydrogenase (ALDH) was discovered on tumor and ascites derived cells from patients diagnosed with ovarian cancer.

CD44 is a surface molecule which mediates cell adhesion and migration by binding extracellular matrix components such as hyaluronic acid, osteopontin, or activating receptor tyrosine kinases, which are linked with tumor progression and metastasis. It has been demonstrated that CD44+ cells with the properties of ovarian CSCs/ TICs are often present in epithelial ovarian cancer presenting as physically powerful risk factors for chemotherapeutic drug resistance in ovarian carcinoma patients [4]. Also, recent studies revealed that the CD44+/CD24- cells found within primary ovarian cancer or ascites are associated with an increased invasion, risk of recurrence and shorter progression-free survival [5].

Beside its role in cancer initiating cells from primary human tumors, CD117 has been used as stem cell marker for identification and characterization of hematopoietic, cardiac and other mesenchymal stem cells. CD117, known also as c-kit, is a type III receptor tyrosine kinase engaged various cellular processes including cell signal transduction; apoptosis; cell differentiation; proliferation; and cell adhesion. Recently, high expression level of CD117 was observed in ovarian cancers and a few CD117+ ovarian cancer cells have shown the ability to self-renew, differentiate, and regenerate tumor in xenograft model. What's more, it has been proposed that CD117 associates with poor response to chemotherapy in ovarian carcinoma [6].

The pentaspan transmembrane glycoprotein CD133, known as Prominin-1, was originally described as a hematopoietic stem cell marker and was then shown to be expressed by progenitor cells of the epithelium. Two types of CD133 surface protein (CD133-1 and CD133-2) were associated with ovarian cancer cells with stem-like phenotype being detected in primary ovarian carcinomas, as well as in normal ovaries and ovarian metastases but with significantly lower percentages [7]. In xenografts, CD133+ cells obtained from ovarian tumors were capable of self-renewal and were associated with increased tumor hostility and contributed to establishment of tumor vasculature, by means of in vivo serial transplantations [8]. In addition, CD133+ ovarian CSCs had hyperactivity in migration and invasion due to the activation of chemokine (c-c motif) ligand 5 (CCL5) [9].

In neoplasia, CD24 expression has been described initially in hematological malignancies but also in a wide array of solid tumors. It is a small, heavily glycosylated mucin-like glycosylphosphatidylinositol (GPI)-linked cell surface protein, which is physiologically expressed in developing or regenerating tissue including granulocytes, pre-Bcells, keratinocytes, and renal tubular epithelium. CD24 is a ligand of P-selectin, an adhesion receptor on activated endothelial cells and platelets, and this might explain the metastasizing capacities of CD24expressing tumor cells [10]. Kristiansen et al. [11] observed dual CD24 polarized membranous and cytoplasmic staining reflecting an overproduction or a disturbance of protein distribution or degradation within invasive ovarian carcinomas but not in normal ovaries and ovarian adenomas and this pattern was a strong and independent predictor of short overall survival. Moreover, CD24+ cells successfully isolated from ovarian tumor specimens possessed stem-like properties, including higher levels of chemoresistance and self-renewal [12].

Recently, human B-cell specific moloney leukemia virus insertionsite1 (Bmi-1), a member of the polycomb group gene (PcG) protein family of transcription repressors, has emerged as a Myc-cooperating oncogene in murine lymphomas that plays a crucial role in cancer development and recurrence. In human, Bmi-1 gene localizes on short arm of chromosome 10 and encodes a protein of $36.8 \mathrm{kDa}$. This protein controls the cell cycle and promotes cell proliferation by suppressing p16INK4a/retinoblastoma and/or p14ARF/MDM2/ p53 tumor suppressor pathways [13]. Likewise, Bmi-1 was reported to maintain self-renewal and differentiation capacities and prevent cellular senescence through the activation of telomerase in adult stem cells, in addition to its significant role in chemoresistance and distant metastasis [14]. In ovarian cancer, it is speculated that Bmi-1 acts as an oncoprotein that might contribute to the carcinogenesis. It was

*Corresponding author: Department of Pathology, Faculty of Medicine, Mansoura University, El-Gomhouria street, Mansoura-Dakahlia, P.O Box 35516, Egypt, Tel: 966557662665; E-mail: amalabdelhafez@gmail.com

Received February 24, 2014; Accepted February 24, 2014; Published February 26, 2014

Citation: Abd El hafez A (2014) Stemness Markers in Ovarian Cancer: Nature and Implications. J Cytol Histol 5: e112. doi:10.4172/2157-7099.1000e112

Copyright: @ $2014 \mathrm{Abd}$ El hafez A. This is an open-access article distributed under the terms of the Creative Commons Attribution License, which permits unrestricted use, distribution, and reproduction in any medium, provided the original author and source are credited. 
immuno-histochemically expressed in a high percentage of ovarian cancers in association with high histological grade, aggressive histology, advanced stage and reduced patient survival. For that reason, Bmi-1 can be used as a marker to separate ovarian cancer patients with more aggressive features, and poor outcome [15].

Increasing evidence showed that, ABCG2/BCRP1(breast cancerresistance protein-1), an ATP-binding cassette (ABC) broad-specificity transmembrane transporter, is a cell surface drug-resistance marker, which has been used to identify stem cells from a variety of tissues, including tumors [16]. This marker was found to be overexpressed by ovarian cancer stem cells being localized at the cancer stem-like cell niche where it is responsible for maintaining asymmetric division; high tumorigenicity and drug resistance for these cells $[17,18]$.

In association to CD133; aldehyde dehydrogenase (ALDH); a reported cancer stem cell marker in various solid tumors, has been investigated with the purpose of recognizing a set of markers to identify ovarian CSCs/TICs. Aldehyde dehydrogenase-1A1 (ALDH1A1) is an intracellular enzyme that oxidizes aldehydes. It has a detoxifying role and allows the conversion of retinol to retinoic acid, thus organizes cellular differentiation pathways. ALDH1A1 was associated with chemoresistance in the ovarian cancer [14], and the presence of dual CD133/ALDH coexpressing cells in debulked primary tumor specimens correlated with reduced disease-free and overall survival in ovarian cancer patients [19], and evidenced superior tumor initiating capability in ovarian cancer cell lines [8].

Therefore, during treatment for ovarian cancer, a tailored chemotherapeutic schedule should be offered taking into account the clonal heterogeneity of ovarian tumor cells and depending on the cellular make-up of each ovarian tumor. Furthermore, maintenance therapy must target the surviving CSCs/TICs aiming to avoid tumor recurrence and improve the survival rate of ovarian cancer.

\section{References}

1. Agarwal R, Kaye SB (2005) Prognostic factors in ovarian cancer: how close are we to a complete picture? Ann Oncol 16: 4-6.

2. Di J, Duiveman-de Boer T, Figdor CG, Torensma R (2013) Aiming to immune elimination of ovarian cancer stem cells. World J Stem Cells 5: 149-162.

3. Mor G, Alvero A (2013) The duplicitous origin of ovarian cancer. Rambam Maimonides Med J 4: e0006.

4. Hu Z, Gao J, Zhang D, Liu Q, Yan L, et al. (2013) High expression of Lewis y antigen and CD44 is correlated with resistance to chemotherapy in epithelial ovarian cancers. PLoS One 8: e57250.
5. Meng E, Long B, Sullivan P, McClellan S, Finan MA, et al. (2012) CD44+/CD24ovarian cancer cells demonstrate cancer stem cell properties and correlate to survival. Clin Exp Metastasis 29: 939-948.

6. Luo L, Zeng J, Liang B, Zhao Z, Sun L, et al. (2011) Ovarian cancer cells with the CD117 phenotype are highly tumorigenic and are related to chemotherapy outcome. Exp Mol Pathol 91: 596-602.

7. Ferrandina G, Bonanno G, Pierelli L, Perillo A, Procoli A, et al. (2008) Expression of CD133-1 and CD133-2 in ovarian cancer. Int J Gynecol Cancer 18: 506-514.

8. López J, Valdez-Morales FJ, Benítez-Bribiesca L, Cerbón M, Carrancá AG (2013) Normal and cancer stem cells of the human female reproductive system. Reprod Biol Endocrinol 11: 53.

9. Long H, Xie R, Xiang T, Zhao Z, Lin S, et al. (2012) Autocrine CCL5 signaling promotes invasion and migration of CD133+ ovarian cancer stem-like cells via NF-Î'B-mediated MMP-9 upregulation. Stem Cells 30: 2309-2319.

10. Welsh JB, Zarrinkar PP, Sapinoso LM, Kern SG, Behling CA et al. (2001) Analysis of gene expression profiles in normal and neoplastic ovarian tissue samples identifies candidate molecular markers of epithelial ovarian cancer. Proc Natl Acad Sci USA 98: 1176-1181.

11. Kristiansen G, Denkert C, Schlüns K, Dahl E, Pilarsky C, et al. (2002) CD24 is expressed in ovarian cancer and is a new independent prognostic marker of patient survival. Am J Pathol 161: 1215-1221.

12. Gao MQ, Choi YP, Kang S, Youn JH, Cho NH (2010) CD24+ cells from hierarchically organized ovarian cancer are enriched in cancer stem cells. Oncogene 29: 2672-2680.

13. Jacobs JJ, Kieboom K, Marino S, DePinho RA, van Lohuizen M (1999) The oncogene and Polycomb-group gene bmi-1 regulates cell proliferation and senescence through the ink4a locus. Nature 397: 164-168.

14. Glinsky GV, Berezovska O, Glinskii AB (2005) Microarray analysis identifies a death-from-cancer signature predicting therapy failure in patients with multiple types of cancer. J Clin Invest 115: 1503-1521.

15. Abd El Hafez A, El-Hadaad HA (2014) Immunohistochemical expression and prognostic relevance of $\mathrm{Bmi}-1$, a stem cell factor, in epithelial ovarian cancer Ann Diagn Pathol 18 (2): 58-62.

16. Diestra JE, Scheffer GL, Catala I, Maliepaard M, Schellens JH et al. (2002) Frequent expression of the multi-drug resistance-associated protein BCRP/ MXR/ABCP/ABCG2 in human tumors detected by the BXP-21 monoclonal antibody in paraffin-embedded material. J Pathol 198: 213-219.

17. Hu L, McArthur C, Jaffe RB (2010) Ovarian cancer stem-like side-population cells are tumourigenic and chemoresistant. Br J Cancer 102: 1276-1283.

18. Luo LJ, Zhao Z, Zeng JF, Liang B, Yang JX, et al. (2012) [Analysis of the characteristics of side population cells in the human ovarian cancer cell line OVCAR-3]. Zhonghua Fu Chan Ke Za Zhi 47: 281-285.

19. Silva IA, Bai S, McLean K, Yang K, Griffith K, et al. (2011) Aldehyde dehydrogenase in combination with CD133 defines angiogenic ovarian cancer stem cells that portend poor patient survival. Cancer Res 71: 3991-4001. 